\title{
SIMULATION OF SECONDARY ELECTRON YIELDS FROM THIN METAL FOILS AFTER FAST PROTON IMPACT
}

\author{
A. Travia and M. Dingfelder* \\ Department of Physics, East Carolina University, Mailstop \#563, Greenville, NC 27858, USA \\ *Corresponding author: dingfelderm@ecu.edu
}

\begin{abstract}
Differential and total inelastic cross sections are derived for the interaction between fast protons and $\mathrm{Cu}$. The calculations are done under the non-relativistic plane-wave first-Born approximation and the dielectric theory. A semi-empirical optical oscillator strength density function and a simple linear-momentum dispersion algorithm are used to construct the energy loss function or Bethe surface of the medium. A transport model using these inelastic cross sections is implemented in the Monte Carlo code PARTRAC to simulate the spectra of secondary electron emissions from this homogeneous and isotropic thin copper foil target. Comparisons with experimental results show general agreement for impact energies $>50 \mathrm{eV}$ up to non-relativistic values. The model, however, overestimates the secondary electron yields at lower energies.
\end{abstract}

\section{INTRODUCTION}

Monte Carlo (MC) codes rely on differential and total collision cross sections to simulate the track structures of particles as they interact with and pass through matter. The detailed event-by-event simulation generally follows the incident primary particle and all produced secondary particles from starting or ejection energies down to total stopping and records all relevant physical quantities to describe the spatial distribution of events and energy depositions in the material under consideration. The accuracy of the simulation is mainly dictated by the quality of the corresponding cross sections used for each considered collision channel ${ }^{(1,2)}$.

The exact calculation of the physical quantities that describe the interaction of charged particles with condensed-phase materials is a formidable task. In this case, the instantaneous multiple interactions between the large number of particles of the medium cannot be neglected. This results in a many-body problem that is impossible to be precisely solved. Therefore, approximations are adopted to permit calculations to be practical and to aid further investigations. This cyclic approach leads to progress towards more realistic outcomes.

Collision experiments involving condensed-phase targets are in general very difficult to perform, especially with low-energy electrons in condensedphase materials of biological interest (e.g. liquid water). However, electron emissions from thin foils (e.g. $\mathrm{Al}, \mathrm{Cu}, \mathrm{Au}$ and those with thin layers of gases like water frozen on them) after fast proton or ion impact can provide valuable information on the lowenergy electron transport within that material. Comparisons of simulation results using MC track structure codes and experimental data can help to evaluate and update implemented transport models and interaction cross sections ${ }^{(3)}$. Metal foils are of special interest since both theoretical calculations and experimental procedures are thought to be better understood than complex biological materials like amorphous solid water (ASW). With this motivation, a simple transport model for electrons and fast protons in copper has been developed and implemented into the MC track structure code PARTRAC. Interaction cross sections have been obtained using a simple non-relativistic projectiletarget interaction model based on first-Born approximation and the dielectric response theory.

\section{INTERACTION CROSS SECTIONS}

First-Born approximation and the dielectric theory

In principle, there are two methods used to derive inelastic differential collision cross sections: the microscopic or full quantum mechanics (FQM) approach and the macroscopic dielectric theory (DT). The FQM approach generally proceeds by constructing the Hamilton operator of the system. Eigenfunctions can be calculated, optimised and linked to the inelastic collision form-factor of the target by solving the Schrödinger equation. The commonly used approximation scheme includes first-Born approximation and the Hartree-Fock selfconsistent method. On the other hand, the macroscopic or DT formalism explains the perturbation caused by the incident charged particle on the condensed-phase target by analysing the dielectric response of the medium caused by this perturbation $^{(4)}$. The resulting key quantity of the DT is called the energy loss function (ELF) or Bethe surface of the target which provides the complete profile of the interaction in energy transfer $w$ and linear-momentum transfer $q$ space. The ELF $\eta_{2}$ is 
given by

$$
\eta_{2}(w, q)=\Im\left[\frac{-1}{\varepsilon(w, q)}\right]=\frac{\varepsilon_{2}(w, q)}{\varepsilon_{1}^{2}(w, q)+\varepsilon_{2}^{2}(w, q)},
$$

where $\varepsilon(w, q)=\varepsilon_{1}(w, q)+\mathrm{i} \varepsilon_{2}(w, q)$ is the (complex) dielectric response function of the medium. $J(z)$ indicates the imaginary part of the complex number $z$.

If the incident charged particle is sufficiently fast with respect to the average velocity of the target electrons, the interaction can be viewed as a sudden and small perturbation. In this case, the most commonly used approximation is (non-relativistic) plane-wave first-Born approximation (PWBA) $)^{(1,2)}$ that greatly simplifies the calculations. Within the PWBA, the doubly differential inelastic macroscopic cross section (DDICS) factorises into two independent parts. The first factor describes exclusively the kinematics of the projectile and is independent of the target material while the second factor completely describes the collision interaction properties of the target for all incoming projectiles. Therefore, within the PWBA and the DT the DDICS is expressed as

$$
\frac{\mathrm{d}^{2} \Sigma}{\mathrm{d} w \mathrm{~d} q}(E ; w, q)=\frac{1}{\pi a_{0}} \frac{1}{E} \frac{1}{q} \eta_{2}(w, q)
$$

where $E$ is the total kinetic energy of the projectile and $a_{0}=0.529 \AA$ the Bohr radius. The macroscopic total inelastic cross section $\Sigma$, which is also known as inverse mean free path (IMFP) of the particle, is related to the microscopic cross section $\sigma$ by $\Sigma=N \sigma$ with $N$ representing the particle density of the medium. Also, the (macroscopic) ELF can be related to the (microscopic) generalised oscillator strength distribution $\mathrm{d} f / \mathrm{d} w$ of the target by

$$
\eta_{2}(w, q)=\frac{\pi}{2} \frac{E_{p}^{2}}{Z} \frac{1}{w} \frac{\mathrm{d} f(w, q)}{\mathrm{d} w}
$$

where $E_{p}^{2}=28.816(\rho Z / A)^{1 / 2}$ is the nominal plasma energy in electron volts, $Z$ the atomic number of the target, $A$ the atomic mass and $\rho$ the mass density.

The single differential IMFP (DIMFP) and the total IMFP are then obtained by successive integration of Equation (2) by considering kinematical requirements:

$$
\frac{\mathrm{d} \Sigma}{\mathrm{d} w}(E ; w)=\int_{q_{\max }}^{q_{\max }} \frac{\mathrm{d}^{2} \Sigma}{\mathrm{d} w \mathrm{~d} q} \mathrm{~d} q, \quad \Sigma(E) \int_{0}^{w_{\max }} \frac{\mathrm{d} \Sigma}{\mathrm{d} w} \mathrm{~d} w .
$$

Integration limits are defined by kinematical considerations and are given by

$$
q_{\min / \max }=\sqrt{2 m}(\sqrt{E} \pm \sqrt{E-w})
$$

where $m$ is the mass of the projectile. The maximum energy transfer is $w_{\max }=E$. In the case of electron impact, exchange effects should be considered; in this case, the maximum energy transfer is limited to $w_{\max }=E / 2$.

\section{The binary description of the Bethe surface of copper}

The ELF of copper is modelled using Equation (3), optical oscillator strength (OOS) data from the literature and a simple extension algorithm, a method also known as optical data model. The OOS taken from the literature ${ }^{(5)}$ is based on Palik's compilation of data ${ }^{(6)}$ and photo-ionisation cross sections ${ }^{(7)}$. The extension algorithm is based on the $\delta$-oscillator model initially introduced by Ashley $^{(8)}$ for proton impact and, for example, reviewed for electron impact in ref. (9). This simple linear-momentum dispersion algorithm treats the interaction as a binary encounter by concentrating the OOS along the $w=q$ binary line, which is also known as the Bethe ridge of the surface. It is expressed as

$$
w \eta_{2}(w, q)=\int_{0}^{w} w^{\prime} \eta_{2}\left(w^{\prime}, 0\right) \delta\left(w^{\prime}-\frac{q^{2}}{2 m}\right) \mathrm{d} w^{\prime} .
$$

The DDICS, Equation (2) can then be expressed using the OOS and Equation (6) as

$$
\frac{\mathrm{d}^{2} \Sigma}{\mathrm{d} w \mathrm{~d} q}(E ; w, q)=\frac{1}{2 a_{0} Z} \frac{E_{p}^{2}}{E} \frac{1}{w q} \frac{\mathrm{d} f\left(w-\left(q^{2} / 2 m\right)\right)}{\mathrm{d} w} .
$$

\section{Shell separations}

Event-by-event MC track structure codes require detailed information about the energy deposited in a collision and transported away as kinetic energy of secondary electrons. Therefore, it is imperative to know from which subshell the secondary electron originated. The total OOS as taken from the literature ${ }^{(5)}$ has been decomposed into subshells by linearly extrapolating the total OOS beyond the $L$ shell on the log-log scale and then subtracting out the contribution from the $K$ shell and continuing. The following subshells are considered: $K$-shell $(1 s ; 8.979 \mathrm{keV}), L$-shell $(2 s / 2 p$; $0.931 \mathrm{keV}), M$-shell $(3 s / 3 p ; 73.6 \mathrm{eV})$ and outer-shell $(4 s / 3 d)$ ionisation and excitations (electronic and plasmon). The total OOS and the contributions from the considered subshells are displayed in Figure 1.

\section{IMFPs for electrons and protons in copper}

Total and energy differential inelastic interaction cross sections have been calculated for the electron and proton impact on copper using Equations (4) and (7). In the case of electron impact, exchange effects as described in refs $(1,2)$ have been included. However, no low-energy corrections have 
been applied, or in other words, no contributions beyond the PWBA have been considered. This is unproblematic for the transport of protons because their total energy remains high enough that the PWBA is valid. For electrons, the PWBA is used as a first approximation recognising the fact that pure PWBA cross sections should not be used below $500 \mathrm{eV}^{(1)}$. The IMFPs for electrons and protons are shown in Figures 2 and 3, respectively.

In both cases contributions from the subshells (excitations and outer shell and $M$-shell), are shown. Contributions from the $L$ - and $K$-shells are too small to be displayed on the current scale. Furthermore, protons ( 2 and $6 \mathrm{MeV}$ ) and produced secondary

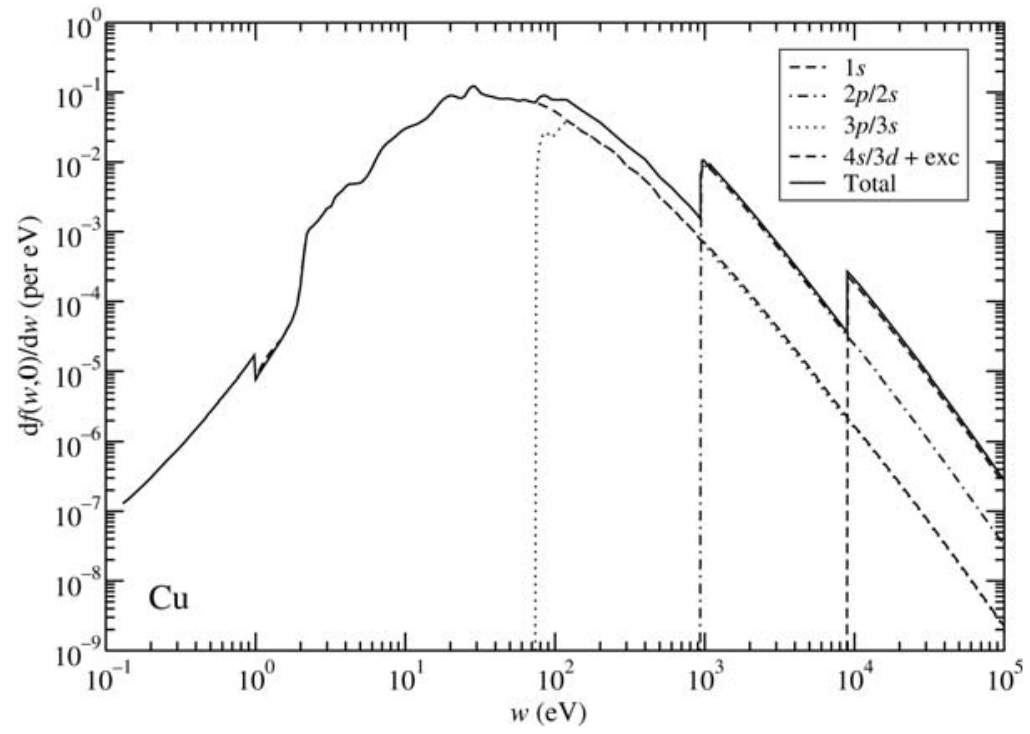

Figure 1. OOS of copper. The total OOS as taken from the literature ${ }^{(5)}$ is partitioned into four subshells: excitations and outer-shell ionisation $(4 s / 3 d), M$-shell $(3 p / 3 s), L$-shell $(2 p / 2 s)$ and $K$-shell $(1 s)$.

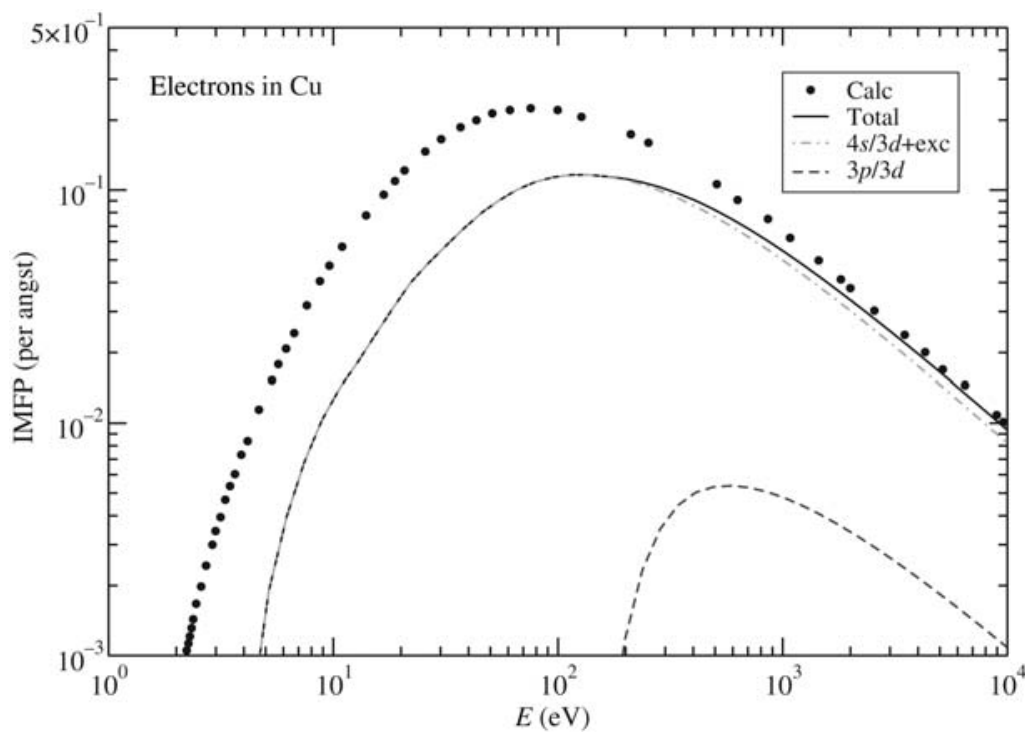

Figure 2. Total IMFPs of electrons in copper. Shown are contributions from the subshells excitations and outer-shell ionisation ( $4 s / 3 d)$, and $M$-shell $(3 p / 3 s)$, and a calculation by Fernández-Varea ${ }^{(9)}$. 


\section{A. TRAVIA AND M. DINGFELDER}

electrons used in this study barely have the energy to ionise the $K$-shell. Figure 2 also displays a calculation for the IMFP of electrons in copper by FernándezVarea et al. ${ }^{(9)}$. These authors use a modified $\delta$-oscillator model and show that the pure $\delta$-oscillator as used in this study does not reproduce a correct threshold behaviour, i.e. tend to higher ionisation thresholds. Figure 4 illustrates DIMFPs for the electron impact on copper for three different incident energies: $100 \mathrm{eV}, 1$ and $10 \mathrm{keV}$, respectively.

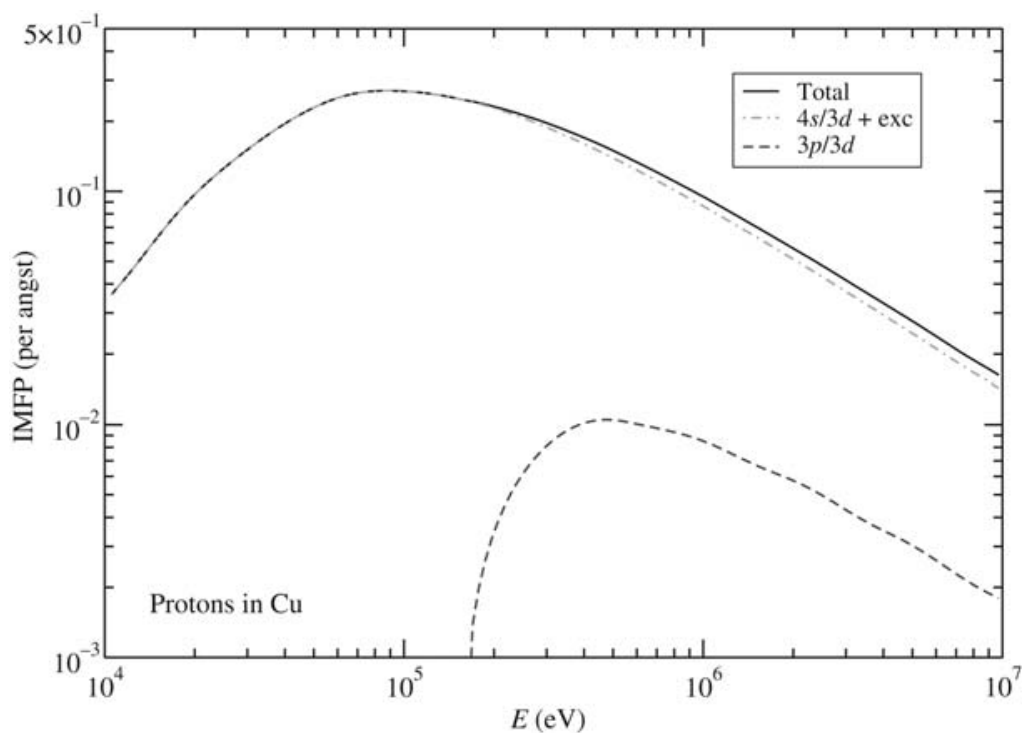

Figure 3. Total IMFPs of protons in copper. Shown are contributions from the subshells excitations and outer-shell ionisation $(4 s / 3 d)$, and $M$-shell $(3 p / 3 s)$.

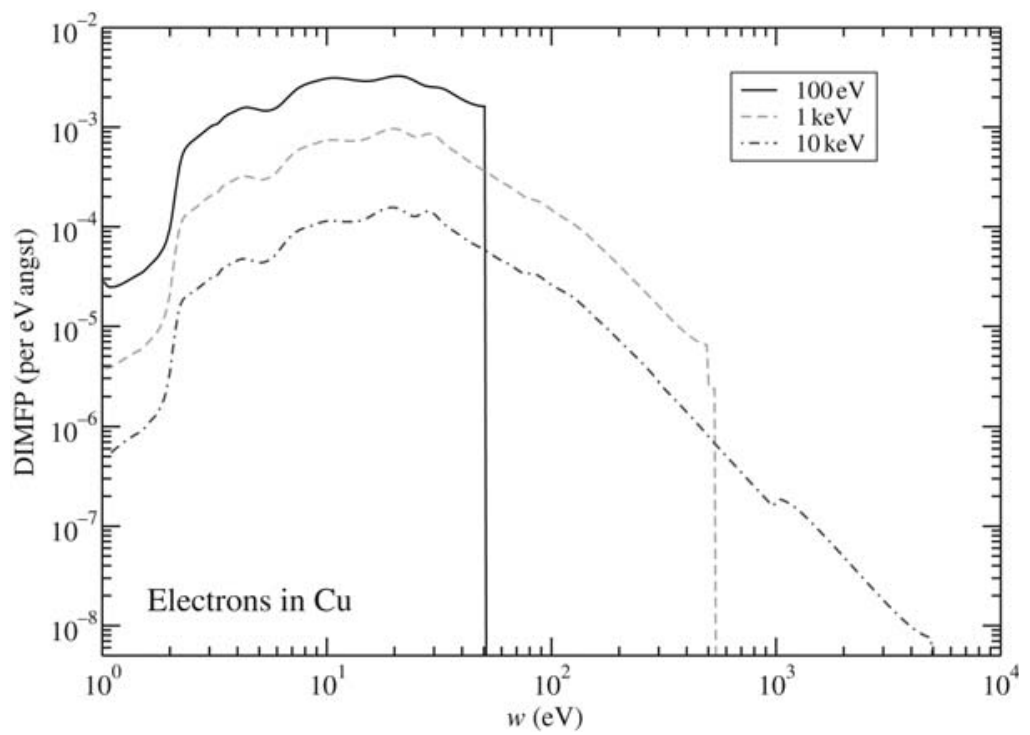

Figure 4. Total DIMFPs for electrons in copper. Shown are DIMFPs for total electron energies of $100 \mathrm{eV}, 1$ and $10 \mathrm{keV}$, respectively. 


\section{SIMULATIONS AND PRELIMINARY RESULTS}

\section{Copper transport model}

A model for the transport of electrons $(1 \mathrm{eV}-10$ $\mathrm{keV})$ and fast protons $(500 \mathrm{keV}-10 \mathrm{MeV})$ in copper has been implemented into the event-by-event track structure code PARTRAC. The proton transport model is based on the above presented IMFPs and DIMFPs and considers the four subshells. The outer-shell and excitation subshell leads to an ionisation if the energy transfer $w \geq 20 \mathrm{eV}$, and to excitations if $w<20 \mathrm{eV}$. Elastic scattering for the protons is not considered. Secondary electron emission spectra after proton impact are modelled using angular distributions of liquid water as described in ref. (10). These angular distributions are obtained from a semi-empirical model based on the Bethe approximation.

The electron transport model is also based on the above-described IMFPs and DIMFPs for the electron impact. As in the case of protons, four subshells are considered. The outer-shell and excitation subshell again leads to an ionisation if the energy transfer $w \geq 20 \mathrm{eV}$, and to excitations if $w<20 \mathrm{eV}$. Elastic scattering cross sections for electrons have been calculated using Salvat's ELSEPA code, as described in the ICRU Report $77^{(11)}$, for the energy range down to $10 \mathrm{eV}$. Below $10 \mathrm{eV}$, elastic scattering cross sections are kept constant at the $10 \mathrm{eV}$ values. The threshold for inelastic events is also set at $10 \mathrm{eV}$. Scattering angles for primary electrons are determined within the binary collision theory. Secondary electrons are emitted isotropically for small energy transfers and under $90^{\circ}$ relative to the primary electron (binary collision) for larger energy transfers.

\section{Electron emission spectra}

Secondary electron spectra from a thin $(1 \mu \mathrm{m})$ copper foil after the fast proton $(6 \mathrm{MeV})$ impact have been simulated. Preliminary simulated electron yields (per eV, sr and proton) are shown for selected forward angles $\left(10^{\circ} \pm 5^{\circ}, 40^{\circ} \pm 5^{\circ}\right.$ and $\left.70^{\circ} \pm 5^{\circ}\right)$ in Figure 5. Comparison with experimentally obtained electron yields obtained by the East Carolina University Accelerator Laboratory ${ }^{(12)}$ (a measurement for $35^{\circ}$ forward angle is also displayed in Figure 5) confirms findings from secondary electron yields simulated and measured for $\mathrm{ASW}^{(3,13)}$ : simulated electron yields in general follow the measurements, but clearly overestimate them at energies below $50 \mathrm{eV}$.

\section{Conclusions}

Secondary electron yield measurements for condensed-phase targets are useful to improve MC track structure codes. Low-energy electron transport, especially in condensed-phase materials is still not well understood and remains a matter of interest. More work on special topics needs to be done, especially on the low-energy transport model and

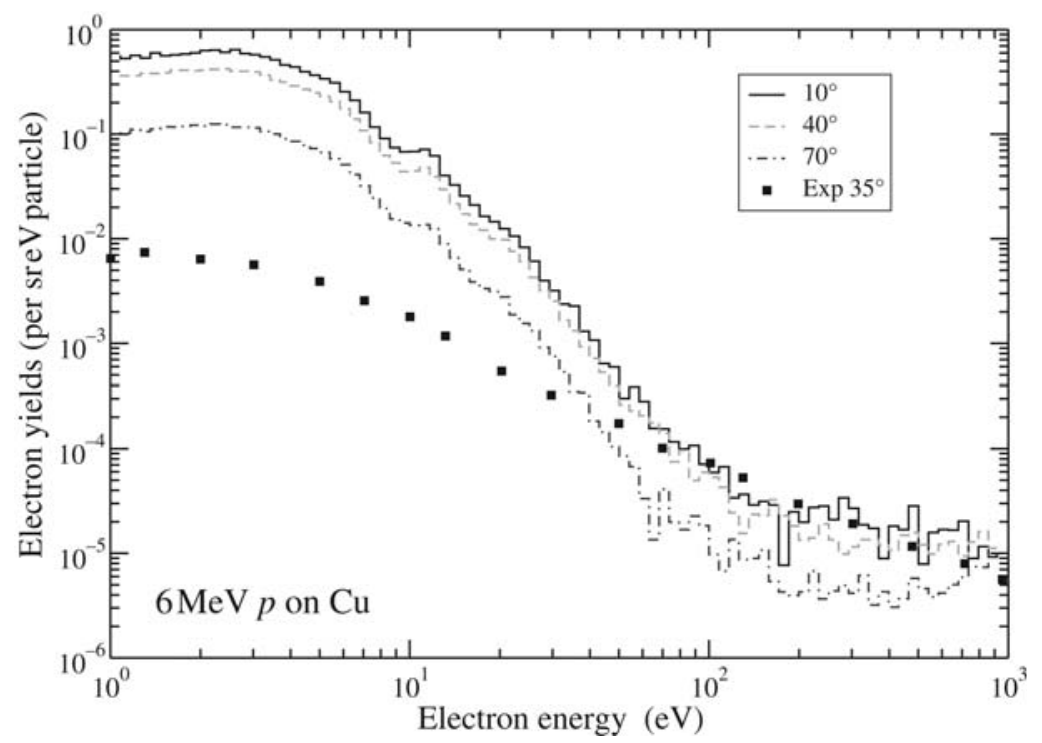

Figure 5. Simulated electron emission yields from copper after a $6-\mathrm{MeV}$ proton impact. Shown are forward angles $10^{\circ}$, $40^{\circ}$ and $70^{\circ}$. Also shown is a measurement ${ }^{(12)}$ for a forward angle of $35^{\circ}$. 


\section{A. TRAVIA AND M. DINGFELDER}

inelastic cross sections (below $20 \mathrm{eV}$ ), and on elastic scattering in the condensed phase, where interesting new work $^{(14)}$ becomes slowly available.

\section{FUNDING}

This work is supported in part by the National Institutes of Health, National Cancer Institute Grant No. 2R01CA093351-04A1.

\section{REFERENCES}

1. Dingfelder, M., Hantke, D., Inokuti, M. and Paretzke, H. G. Electron inelastic-scattering cross sections in liquid water. Radiat. Phys. Chem. 53, 1-18 (1998).

2. Dingfelder, M., Ritchie, R. H., Turner, J. E., Friedland, W., Paretzke, H. G. and Hamm, R. N. Comparisons of calculations with PARTRAC and NOREC: transport of electrons in liquid water. Radiat. Res. 169, 584-594 (2008).

3. Dingfelder, M., Travia, A., McLawhorn, R. A., Shinpaugh, J. L. and Toburen, L. H. Electron emission from foils and biological materials after proton impact. Radiat. Phys. Chem. 77, 1213-1217 (2008).

4. Hubbard, J. The dielectric theory of electronic interactions in solids. Proc. Phys. Soc. A 68, 976-986 (1955).

5. Fernández-Varea, J. M., Salvat, F., Dingfelder, M. and Liljequist, D. A relativistic optical-data model for inelastic scattering of electrons and positrons in condensed matter. Nucl. Instrum. Methods Phys. Res. B 229, 187-218 (2005).

6. Palik, E. D. Handbook of Optical Constants in Solids. Academic Press (1985).
7. Cullen, D. E., Hubbell, J. H. and Kissel, L. EPDL97 the evaluated data library, '97 version. Report UCRL50400, Vol. 6, Rev. 5. Lawrence Livermore National Laboratory (1997).

8. Ashley, J. C. Optical model for the stopping power of condensed matter for protons and antiprotons. J. Phys. Condens. Matter 3, 2741-2753 (1991).

9. Fernández-Varea, J. M., Mayol, R., Salvat, F. and Liljequist, D. A comparison of inelastic electron scattering models based on $\delta$-function representations of the Bethe surface. J. Phys. Condens. Matter 4, 2879-2890 (1992).

10. Dingfelder, M. Cross section calculations in condensed media: charged particles in liquid water. Radiat. Prot. Dosim. 99, 23-28 (2001).

11. International Commission on Radiation Units and Measurements (ICRU). Elastic scattering of electrons and positrons. ICRU Report 77. Oxford University Press (2007)

12. McLawhorn, S. L., McLawhorn, R. A., Carnes, K. D., Richard, P., Dingfelder, M., Toburen, L. H. and Shinpaugh, J. L. Doubly differential electron yields from thin copper foils induced by fast proton impact. In: CP 1099, Application of Accelerators in Research and Industry: 20th International Conference. McDaniel, F. D. and Doyle, B. L., Eds. (American Institute of Physics) pp. 176-179 (2009).

13. Toburen, L. H., McLawhorn, S. L., McLawhorn, R. A., Carnes, K. D., Dingfelder, M. and Shinpaugh, J. L. Electron emission from amorphous solid water induced by passage of energetic protons and fluorine ions. Radiat. Res. 174, 107-118 (2010).

14. Bote, D., Salvat, F., Jablonski, A. and Powell, C. J. The effect of inelastic absorption on the elastic scattering of electrons and positrons in amorphous solids. J. Electron Spectrosc. Relat. Phenom. 175, 41-54 (2009). 